\title{
Getting peptide vaccines to work: just a matter of quality control?
}

\author{
Esteban Celis \\ Department of Immunology, Mayo Clinic, Rochester, Minnesota, USA \\ J. Clin. Invest. 110:1765-1768 (2002). doi:10.1172/JCI200217405.
}

\section{Optimizing peptide vaccine composition}

Synthetic peptide-based vaccines, which are designed to elicit $\mathrm{T}$ cell immunity, are an attractive approach to the prevention or treatment of infectious diseases and malignant disorders (1-4). CTLs recognize antigens as small protein fragments of eight to ten residues (known as peptide epitopes) that associate with MHC molecules, which are expressed on the surface of antigen-presenting cells (APCs) such as infected or malignant cells (5). As the result of the specific interaction between the $\mathrm{T}$ cell receptor for antigen (TCR) and the peptide/MHC complexes, the CTLs are able to kill target cells expressing infectious or tumorderived antigens. In consequence, manufacturing these $T$ cell epitopes by conventional organic peptide synthesis methods is a relatively simple, expedient, and cost-effective way to produce vaccines for any type of disease in which CTLs could play a role in the elimination of the offending agent.

Unfortunately, the advantages that peptide vaccines have to offer are to some extent diminished by their inherent lack of immunogenicity, which so far has been reflected by their not-sospectacular results in the clinic. Because the immune system in most species has evolved through time to fight life-threatening infectious agents

\footnotetext{
Address correspondence to: Esteban Celis, Department of Immunology, Mayo Clinic, GU-421A, Rochester, Minnesota 55905, USA. Phone: (507) 284-0124; Fax: (507) 266-5255; E-mail: celis@mayo.edu.

Conflict of interest: The author has declared that no conflict of interest exists.

Nonstandard abbreviations used: antigenpresenting cell (APC); human leukocyte antigen (HLA); transporter associated with antigen processing (TAP); endoplasmic reticulum (ER).
}

(and perhaps tumors), it should not be surprising that vaccines consisting of aseptic, endotoxin-free peptides are likely to be ignored and will likely be ineffective at inducing $\mathrm{T}$ cell immunity. In addition, peptides that are injected in aqueous solutions will be unsuccessful at stimulating CTL responses, either because of their rapid biodegradation (e.g., by proteases) or, worse, because of the induction of T cell tolerance/anergy, which results from the antigenic stimulation of CTLs by nonprofessional APCs $(6,7)$. A means for enhancing the immunogenicity of synthetic peptide vaccines has been the use of adjuvants, which aim at mimicking the danger signals produced by pathogens, provoking inflammatory reactions that awaken the immune system (8). In addition, some adjuvants also create a depot effect, preventing access of the immunogen to proteases and allowing their slow release into the extracellular compartments. Thus, it is hoped that peptides that are appropriately formulated in adjuvants can elicit CTL responses that in some instances will have positive effects against infections or tumors.

An additional complication resulting from the use of synthetic peptide-derived vaccines is the induction of CTLs that, while capable of killing target cells that are exogenously pulsed with peptide, are not able to recognize target cells that naturally process and present the peptide epitope, such as infected or malignant cells. Obviously, these "low-quality" CTLs would have little effect in fighting and controlling disease. One of the reasons for the generation of such lowquality CTLs by peptide vaccines is the induction of CTLs with low affinity for antigen, which will require a high density of specific peptide/MHC complexes on the target-cell surface to exert their effector function. In vitro, the induction of low-affinity CTLs usually results from the use of high concentrations of peptide, generating a high level of specific peptide/MHC complexes on APCs, which will effectively activate these CTLs (9). The prediction is that high densities of peptide/MHC complexes on APCs in vivo resulting from an excessive peptide dose will also produce low-quality CTLs with low affinity for antigen.

Another cause for the induction of low-quality CTLs is the use of vaccines produced with synthetic peptides representing cryptic T cell epitopes, which are not expressed on the surface of the infected or tumor target cells (10). The peptides corresponding to cryptic epitopes are immunogenic, because they bind well to MHC molecules on APCs and are able to activate T cells. However, because the same peptide/MHC complexes are not generated through the natural antigen-processing machinery in the infected or tumor cells, CTLs recognizing cryptic epitopes will be unable to interact with infected or tumor target cells and will also be useless in disease control.

\section{Cryptic epitopes alter the immune response}

In this issue of the JCI, Dutoit and collaborators (11) describe the generation of "low-quality" CTL responses in cancer patients vaccinated with two synthetic peptides derived from the sequence of the cancertestis NY-ESO-1 tumor antigen (12). Both of the above-mentioned mechanisms appear to be responsible for this outcome. One of the peptides, NY-ESO-1 $1_{157-165}$, representing a human leukocyte antigen-A2-restricted (HLA-A2-restricted) CTL epitope, while capable of stimulating some CTL responses against tumor cells 


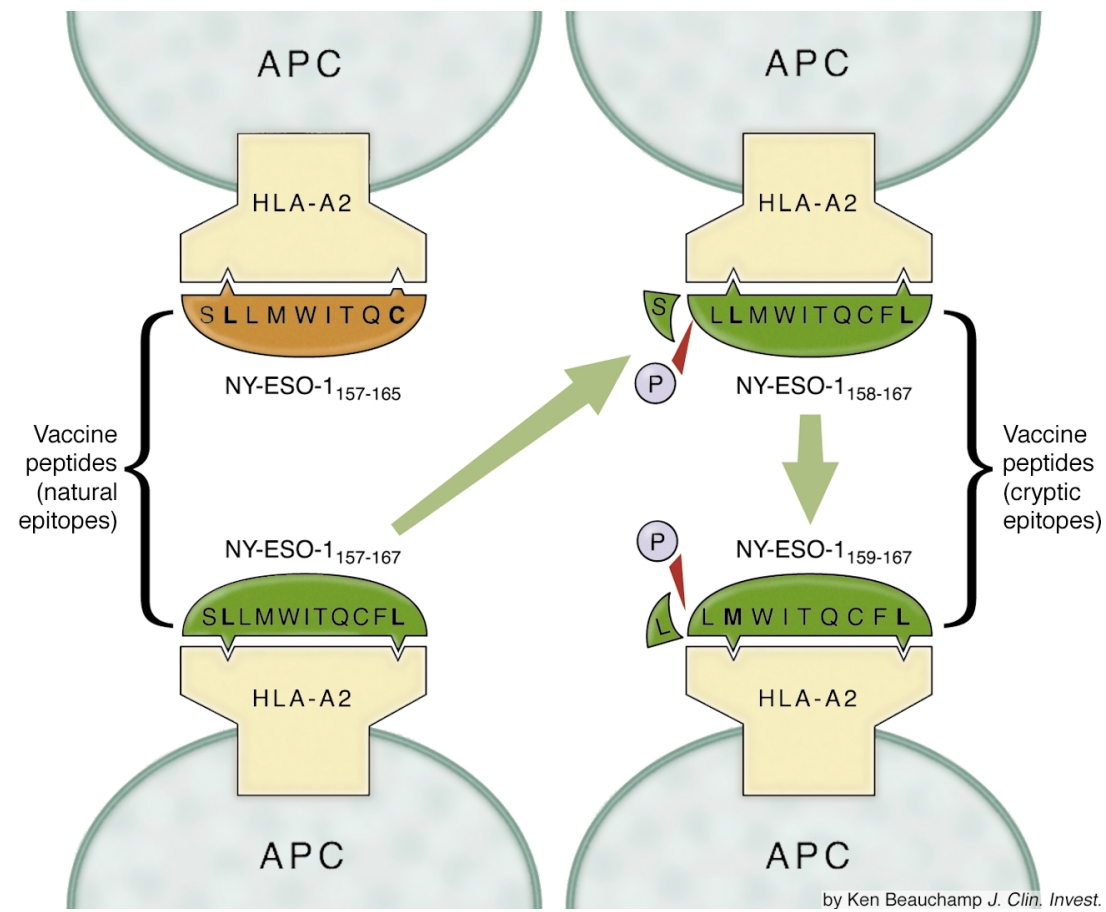

Figure 1

Formation of cryptic CTL epitopes by cleavage of amino-terminal residues in a peptide vaccine. Peptides NY-ESO-1 157-165 and NY-ESO-1 157-167, representing HLA-A2-restricted CTL epitopes, were used to vaccinate cancer patients. By the action of an aminopeptidase (P), peptide NY-ESO-1 $1_{157-167}$ can

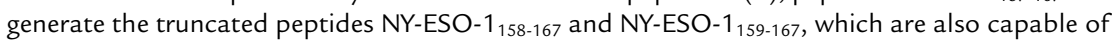
binding to HLA-A2 molecules and were shown to induce low-quality CTLs that failed to recognize tumor cells. Anchor residues, within the peptides, that facilitate $\mathrm{MHC}$ binding are shown in boldface. In the case of NY-ESO-1 $157-165$, the cysteine (C) anchor is likely to be suboptimal, since it does not represent a canonical HLA-A2-binding motif.

expressing the NY-ESO-1 antigen, induced a large number of peptide-reactive CTLs that were incapable of recognizing the tumor cells. These lowquality CTLs exhibited a lower affinity for antigen than the tumor-reactive CTLs. Notably, in this clinical study, the vaccine consisted of $100 \mu \mathrm{g}$ of peptide in solution (formulated in 100\% DMSO), administered with GM-CSF as adjuvant, which presumably attracts professional APCs to the injection site. It is likely that this vaccine formulation would result in the production of APCs with a high density of peptide/ MHC complexes that could lead to the generation of low-affinity CTLs.

The other peptide used in the clinical study by Dutoit et al. (11) was the 11-residue peptide, NY-ESO-1 157-167, that previously was shown to correspond to an HLA-A2-restricted epitope recognized by tumor-reactive CTLs isolated from cancer patients (13). Interestingly, Dutoit et al. show that cancer patients vaccinated with NY-ESO-1 157-167 produced CTLs reactive with two truncated fragments represented by peptides NY-ESO- $1_{158-167}$ and NY-ESO- $1_{159-167}$ (Figure 1). Notably, both of these truncated products of peptide NY-ESO-1 157-167 remained capable of binding well to HLA-A2 molecules. Most disturbing was the finding that the CTLs recognizing the truncated peptides NY-ESO-1 $1_{158-167}$ and NY-ESO-1 $159-167$ could not kill NY-ESO-1-positive tumor cells, indicating that these peptides behave as typical cryptic epitopes. In the discussion of their findings, the authors propose that peptides NY-ESO- $1_{158-167}$ and NY-ESO- $1_{159-167}$ are not produced by the natural (i.e., endogenous) MHC class I antigen-processing pathway, since proteasomal cleavage of NY-ESO-1 is predicted to take place after residue 165, not after residue 167.

One unanswered question is how peptide NY-ESO- $1_{157-167}$ becomes truncated to generate the cryptic (and useless) CTL epitopes. An obvious possibility, explored by the authors (11), is that serum proteases (in this case an aminopeptidase) cleaved one or two residues in peptide NY-ESO- $1_{157-167}$, producing NY-ESO- $1_{158-167}$ and NY-ESO- $1_{159-167}$. However, experimental data presented in their paper do not support this explanation, indicating that peptide NY-ESO- $1_{157-167}$ may be truncated at another site or by a different mechanism. In order to address this issue it would be helpful to examine and understand how peptide-based vaccines generate peptide/MHC complexes on APCs, which are required for CTL activation.

Based on a large number of observations in vitro, most researchers in the field believe that synthetic peptides representing minimal CTL epitopes can bind to cell surface MHC molecules that either are temporarily empty or bear endogenous low-affinity binding peptides, which the exogenously added synthetic peptides are able to displace from the MHC molecule (Figure 2). In this scenario, minimal peptide epitopes do not require internalization or further processing by the APC. It is assumed, but has not been demonstrated, that these in vitro observations would extend to the in vivo scenario resulting from peptide vaccination using minimal epitopes. The question of how peptides larger than the minimal CTL epitope gain access to MHC molecules may be more complex. As mentioned above, in some cases serum proteases are known to trim the large peptides into the minimal MHC-binding CTL epitopes (14). However, in the studies reported by Dutoit et al. (11), this mechanism did not seem to occur with peptide NY-ESO- $1_{157-167}$. Other possible sources of proteases that are involved in extracellular peptide cleaving are the endopeptidases and exopeptidases that are present on the cell surface or secreted by APCs (15). Thus, it is possible that, through the action of these APCderived proteases, extracellular antigen processing of large peptides occurs, generating MHC class I-binding epitopes for CTLs. One strong candidate that may be responsible for processing peptide NY-ESO- $1_{157-167}$ into the cryptic CTL epitopes NY-ESO- $1_{158-167}$ and NY-ESO- $1_{159-167}$ is CD13, which corresponds to a cell surface aminopeptidase that is present on APCs such as monocytes and dendritic cells (16).

Other possibilities can also be considered in the processing and trimming of exogenous large peptides into CTL epitopes within intracellular compart- 
ments, which would require peptide internalization by the APC. It is well known that APCs (monocytes and immature dendritic cells) are highly pinocytic and phagocytic and would be capable of taking up soluble or particulate peptides into their endocytic compartments (Figure 2) $(17,18)$. In some specialized APCs, exogenously acquired proteins or peptides are able to gain access into the cytoplasm, where they incorporate into the MHC class I antigen-processing pathway (a process known as antigen cross-presentation) (19). It is conceivable that if a peptide such as NY-ESO- $1_{157-167}$ gains access to the APC's cytoplasm it could be funneled by the transporter associated with antigen processing (TAP) complex into the endoplasmic reticulum (ER), where resident aminopeptidases could trim some of the peptide (20), generating the MHC-binding NY-ESO- $1_{158-167}$ and NY-ESO-1 $1_{159-167}$ epitopes. Interestingly, peptide NY-ESO-1 157-167 contains a hydrophobic residue at its carboxyl end (a leucine), which is highly suitable for transfer by TAP into the ER (21).

The intracellular processing of large peptides into MHC class I epitopes might occur directly in the endosomal compartments, where resident proteases are capable of trimming these peptides. In this case, the shortened peptides could bind to a subset of MHC class I molecules shown to recycle through the endocytic compartments, where they are capable of exchanging peptides (Figure 2) $(22,23)$.

\section{Possible solutions for the prevention of low-quality CTL responses}

The work presented by Dutoit et al. (11) bears important lessons that may aid in the design and optimization of peptide-based vaccines by helping to avoid the generation of low-quality CTLs. First, it is clear that the induction of low-affinity CTLs could be minimized, perhaps by a more conscientious evaluation of the peptide dose to be used, or by formulating the vaccines in adjuvants that slowly release the peptides to avoid supraoptimal peptide/MHC densities in APCs. Second, a strategy to avoid the use of peptides representing cryptic CTL epitopes could be developed. Such a strategy would dictate that, in the first place, only those peptide epitopes that have been proven to induce CTLs capable of killing infected or tumor target cells should be considered as vaccine candidates. Next, it would be preferable to select peptides that contain no potential cryptic epitopes within their sequence. Since most human CTLs recognize peptides of nine to ten residues, and since one would predict that the shorter the peptide, the less likely that it will generate a cryptic epitope, it would be preferable to select 9-mer peptides over 10-mers (or 11-mers such as NY-ESO-1 $1_{157-167}$ ) to include in a vaccine. In some circumstances, peptides larger than nine residues might contain noncryptic CTL epitopes. For example, the 10-mer glycoprotein gp100 177-186 melanoma CTL epitope (ALMGTHTMEV) contains a second embedded epitope (gp100 $178-186$, LMGTHTMEV), but in this case CTLs recognizing either one of these epitopes were capable of killing melanoma tumor cells (24).

Is it possible to predict whether a peptide of more than nine residues would contain within its sequence other epitopes, which could potentially represent cryptic epitopes? With the help of powerful and sophisticated MHC-binding prediction algorithms easily available through the Internet $(25,26)$, one could examine peptide sequences for the presence of potential cryptic epitopes. In fact, using one of these algorithms, Dutoit et al. retrospectively determined that the truncated peptides NY-ESO- $1_{158-167}$ and NY-ESO- $1_{159-167}$ had a high likelihood of binding to HLA-A2 and could stimulate CTL responses (11). However, because these algorithms are not always accurate and cannot predict whether the epitopes would be of the

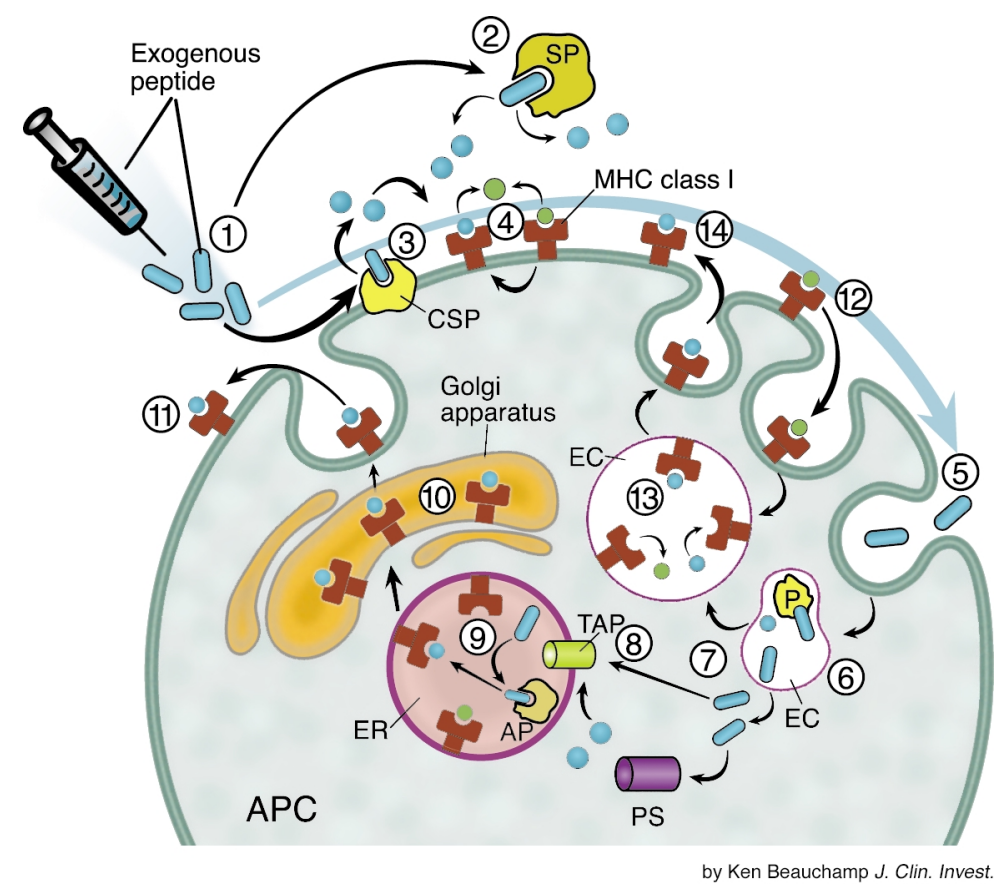

Figure 2

Possible mechanisms to explain how exogenous peptides could be processed to form MHC class I-binding peptides, some of which may constitute cryptic epitopes. Peptides derived from a vaccine (step 1 ) could be cleaved by serum proteases (SP) (step 2 ) or cell surface proteases (CSP) on the APC (step 3 ) to form products capable of binding to cell surface MHC class I molecules that are temporarily empty. Alternatively, the exogenously added peptide representing the minimal CTL epitope may bind to surface $\mathrm{MHC}$ molecules by displacing endogenously-bound peptides (step 4). Endocytosed peptides (step 5) may be cleaved by resident proteases (P) in endosomal compartments (EC) or may gain access to the cytoplasm (step 7) where proteasomes (PS) may cleave them. The cytoplasmic peptides gain access to the endoplasmic reticulum (ER) through the action of the transporter associated with antigen processing (TAP) (step 8). Once in the ER, peptides may be additionally trimmed by resident aminopeptidases (AP), where they bind to empty MHC class I molecules (step 9). The peptide/MHC complexes travel via the Golgi apparatus (step 10) to the cell surface, where they are exposed to $T$ cell interactions (step 11). Peptides that are endocytosed can also bind to MHC class I molecules that recycle through ECs (step 13) to the cell surface (step 14). 
cryptic type, more extensive in vitro experimental work would be needed to ascertain the potential risk of eliciting $\mathrm{T}$ cell responses to cryptic epitopes by peptide vaccination.

The most critical issue that must be addressed is whether the induction of low-quality CTLs, either of low affinity to a natural tumor epitope (such as NY-ESO- $1_{157-165}$ ) or recognizing cryptic epitopes (NY-ESO-1 $158-167$ and NY-ESO-1 159-167), would have a negative impact on the effectiveness of the vaccine against disease. The answer will likely depend on whether the stimulation and expansion of low-quality CTLs have a detrimental effect on the induction of the high-quality, tumorreactive CTLs. For instance, it would be unfortunate if the CTL response to the cryptic epitope dominates the response to the natural epitope (e.g., because it binds better to $\mathrm{MHC}$ ), to the point where little or no response to the natural epitope is detected. This appeared to be the case in the study of Dutoit et al. (11), in which the CTL responses of the vaccinated patients to the natural epitope NY-ESO- $1_{157-167}$ were insignificant compared with the responses to the NY-ESO- $1_{158-167}$ and NY-ESO- $1_{159-167}$ cryptic epitopes. Interestingly, the most dominant of the cryptic epitopes, peptide NY-ESO-1 159-167, was shown to bind to HLA-A2 almost sevenfold better than the natural epitope NY-ESO-1 ${ }_{157-167}$.

In conclusion, although peptide vaccines constitute an attractive path for immunotherapy, there are various issues that must be addressed in order to increase the likelihood that these vaccines will preferentially stimulate high-quality CTL responses and will therefore be effective against disease. Close examination of peptide dose and vaccine formulation, and identification of potential cryptic $T$ cell epitopes, are warranted in the design of future clinical studies.

1. Berzofsky, J.A. 1995. Designing peptide vaccines to broaden recognition and enhance potency. Ann. NY Acad. Sci. 754:161-168.

2. Kast, W.M., and Melief, C.J. 1991. In vivo efficacy of virus-derived peptides and virus-specific cytotoxic $\mathrm{T}$ lymphocytes. Immunol. Lett. 30:229-232.

3. Rothbard, J. 1987. Synthetic peptides as vaccines. Nature. 330:106-107.

4. Buteau, C., Markovic, S.N., and Celis, E. 2002 Challenges in the development of effective peptide vaccines for cancer. Mayo Clin. Proc. 77:339-349

5. Zinkernagel, R.M., and Doherty, P.C. 1979 MHC-restricted cytotoxic T cells: studies on the biological role of polymorphic major transplantation antigens determining T-cell restrictionspecificity, function, and responsiveness. $A d v$. Immunol. 27:51-177.

6. Kyburz, D., et al. 1993. T cell immunity after a viral infection versus $\mathrm{T}$ cell tolerance induced by soluble viral peptides. Eur. J. Immunol. 23:1956-1962.

7. Toes, R.E., Offringa, R., Blom, R.J., Melief, C.J., and Kast, W.M. 1996. Peptide vaccination can lead to enhanced tumor growth through specific T-cell tolerance induction. Proc. Natl. Acad. Sci. USA. 93:7855-7860.

8. Schijns, V.E. 2001. Induction and direction of immune responses by vaccine adjuvants. Crit. Rev. Immunol. 21:75-85.

9. Alexander-Miller, M.A., Leggatt, G.R., and Berzofsky, J.A. 1996. Selective expansion of high or low-avidity cytotoxic T lymphocytes and efficacy for adoptive immunotherapy. Proc. Natl. Acad. Sci. USA. 93:4102-4107.

10. Sercarz, E.E., et al. 1993. Dominance and crypticity of T cell antigenic determinants. Annu. Rev. Immunol. 11:729-766.

11. Dutoit, V., et al. 2002. Multiepitope $\mathrm{CD}^{+} \mathrm{T}$ cell response to an NY-ESO-1 peptide vaccine results in imprecise tumor targeting. J. Clin. Invest. 110:1813-1822. doi:10.1172/JCI200216428.

12. Chen, Y.T., et al. 1997. A testicular antigen aberrantly expressed in human cancers detected by autologous antibody screening. Proc. Natl. Acad. Sci. USA. 94:1914-1918.

13. Jager, E., et al. 1998. Simultaneous humoral and cellular immune response against cancer-testis antigen NY-ESO-1: definition of human histocompatibility leukocyte antigen (HLA)-A2-binding peptide epitopes. J. Exp. Med. 187:265-270.

14. Falo, L.D., Jr., Colarusso, L.J., Benacerraf, B., and Rock, K.L. 1992. Serum proteases alter the antigenicity of peptides presented by class I major histocompatibility complex molecules. Proc. Natl. Acad. Sci. USA. 89:8347-8350.

15. Amoscato, A.A., Prenovitz, D.A., and Lotze, M.T 1998. Rapid extracellular degradation of synthetic class I peptides by human dendritic cells. J. Immunol. 161:4023-4032.

16. Larsen, S.L., Pedersen, L.O., Buus, S., and Stryhn, A. 1996. T cell responses affected by aminopeptidase N (CD13)-mediated trimming of major histocompatibility complex class II-bound peptides. J. Exp. Med. 184:183-189.

17. Banchereau, J., et al. 2000. Immunobiology of dendritic cells. Annu. Rev. Immunol. 18:767-811.

18. Sallusto, F., Cella, M., Danieli, C., and Lanzavecchia, A. 1995. Dendritic cells use macropinocytosis and the mannose receptor to concentrate macromolecules in the major histocompatibility complex class II compartment: downregulation by cytokines and bacterial products. J. Exp. Med. 182:389-400.

19. Carbone, F.R., and Bevan, M.J. 1990. Class Irestricted processing and presentation of exoge nous cell-associated antigen in vivo. J. Exp. Med. 171:377-387.

20. Snyder, H.L., Yewdell, J.W., and Bennink, J.R. 1994. Trimming of antigenic peptides in an early secretory compartment. J. Exp. Med. 180:2389-2394

21. Neefjes, J., et al. 1995. Analysis of the fine specificity of rat, mouse and human TAP peptide transporters. Eur. J. Immunol. 25:1133-1136.

22. Gromme, M., et al. 1999. Recycling MHC class I molecules and endosomal peptide loading. Proc. Natl. Acad. Sci. USA. 96:10326-10331.

23. Chiu, I., Davis, D.M., and Strominger, J.L. 1999. Trafficking of spontaneously endocytosed MHC proteins. Proc. Natl. Acad. Sci. USA. 96:13944-13949.

24. Tsai, V., et al. 1997. Identification of subdominant CTL epitopes of the gp100 melanoma-associated antigen by primary in vitro immunization with peptide-pulsed dendritic cells. J. Immunol. 158:1796-1802.

25. Parker, K.C., Bednarek, M.A., and Coligan, J.E. 1994. Scheme for ranking potential HLA-A2 binding peptides based on independent binding of individual peptide side-chains. J. Immunol. 152:163-175.

26. Rammensee, H., Bachmann, J., Emmerich, N.P., Bachor, O.A., and Stevanovic, S. 1999. SYFPEITHI: database for MHC ligands and peptide motifs. Immunogenetics. 50:213-219. 